\title{
Improving The Intelligent Control Of Magnetic Levitiation Ball Using Artificial Neural Network

\author{
Asogwa Tochukwu Chijindu ${ }^{1}$, Ugwu Edith Angela ${ }^{2}$ \\ ${ }^{1,2}$ Computer Science, Enugu State University of Science and Technology, Enugu, Nigeria
}

\begin{abstract}
Forecasting the time series behavior of magnetic levitation system has been a major research objective for the last five decades. This is due to the challenges presented as a result of its dynamic nature in motion. Initially this problem was supposed to be solves by control engineering researchers using predictive control modeling. However, due to the robust nature of the technological application (high speed train, maglev ball, e.t.c), precision in the control system has been a major challenge according to Earnshaw's theorem. This paper improves intelligent control of a magnetic levitation (ball) system using artificial neural network. First the maglev system plant is identified and then a Neuro controller is designed to predict the dynamic behavior of this system using feedback linearization process. The system will be design and simulated using neural network tool and Mathlab.
\end{abstract}

Keywords: Neuro controller, feedback linearization, magnetic levitation

\section{Introduction}

Over the last fifty years, the use of magnetic and electric fields as a method of actuation has been a research focus. The initial applications of magnetic actuation were in solenoid actuators, magnetic frictionless ball bearing, vehicle brakes, magnetic vibration isolation of sensitive machinery and electrical switches. Approximately twenty years later, the first magnetic levitation train was proposed and initialized as a high speed mass transportation vehicle [1]. Various experiments and large number of theoretical studies have been done in China, Japan, Germany and the United States, all resulting to an interesting results which today have changes market economics and forced manufacturers to develop new technologies for actuation.

Magnetic levitation (maglev) is the use of controlled magnetic fields (magnetic forces) to cause a magnetic object to float in air, countering force of gravity. Most of the current maglev systems are of the electromagnetic suspension (EMS) type, whereby electric current variations control the attractive force of an electromagnet. The mathematical models of such systems are highly nonlinear and open-loop unstable. Hence it is not a trivial task to construct a high performance controller to accurately position the levitated object.

Recently, artificial neural network is the latest technical introduction in the field of control system; this is due to its ability to solve complex non linear problems and exhibits to a very least extent fault characteristics. Hence this intelligent tool will be used to train a neuro controller that controls the maglev system using Mathlab as the implementation tool.

\section{Research Objectives}

i. To predict and control the dynamic behavior of a maglev system using artificial neural network

ii. To design a system that trains and solves time series modeling problem using artificial intelligence (neuro controller)

iii. To improve the linearization and control of maglev system using predictive control model.

iv. To study, design, analyze and implement the system using Mathlab

\section{Literature Review}

In 2007, [2] presented a system linearization model for the control of non linear maglev system. The research model was able to linearize the acquired non linear system using first order terms 
and Fourier series. [3] Researched and proposed a system that employs a phase lead compensator with an open loop pole in the right phase for the stability of a dynamic system within $1.5 \mathrm{~mm}$ response step around the operating point. In [4] Artificial neural network was used as a controller of the retention process. However, the technique has large number of neurons and this delay the processing and training time for a complete controlling process. [5] Was able to control a non linear system combining system linearization and the technique presented in [3], using virtual pole cancellation to design a controller that maintains better system stability in a levitated ball. In 2007, a position control technique for maglev permanent magnet was proposed by [6], using the lyapunov Operation of a Maglev System stability principle to design the controller. The positioning result was good according to their evaluation, although within a range of $30 \mathrm{~mm}$ which is an improvement, but still not very precise in accuracy.

\section{Methodology}

The components for the development of the magnetic levitation system includes the electromagnetic coils, York, sensors, ferromagnetic ball of $\mathrm{m}(\mathrm{g})$, power supply $(\mathrm{dc})$ and neuro controller. Most of these components are already discussed in previous works cited in our literature reviewed; however we will focus our discussion on the principle of operation of the proposed maglev system.

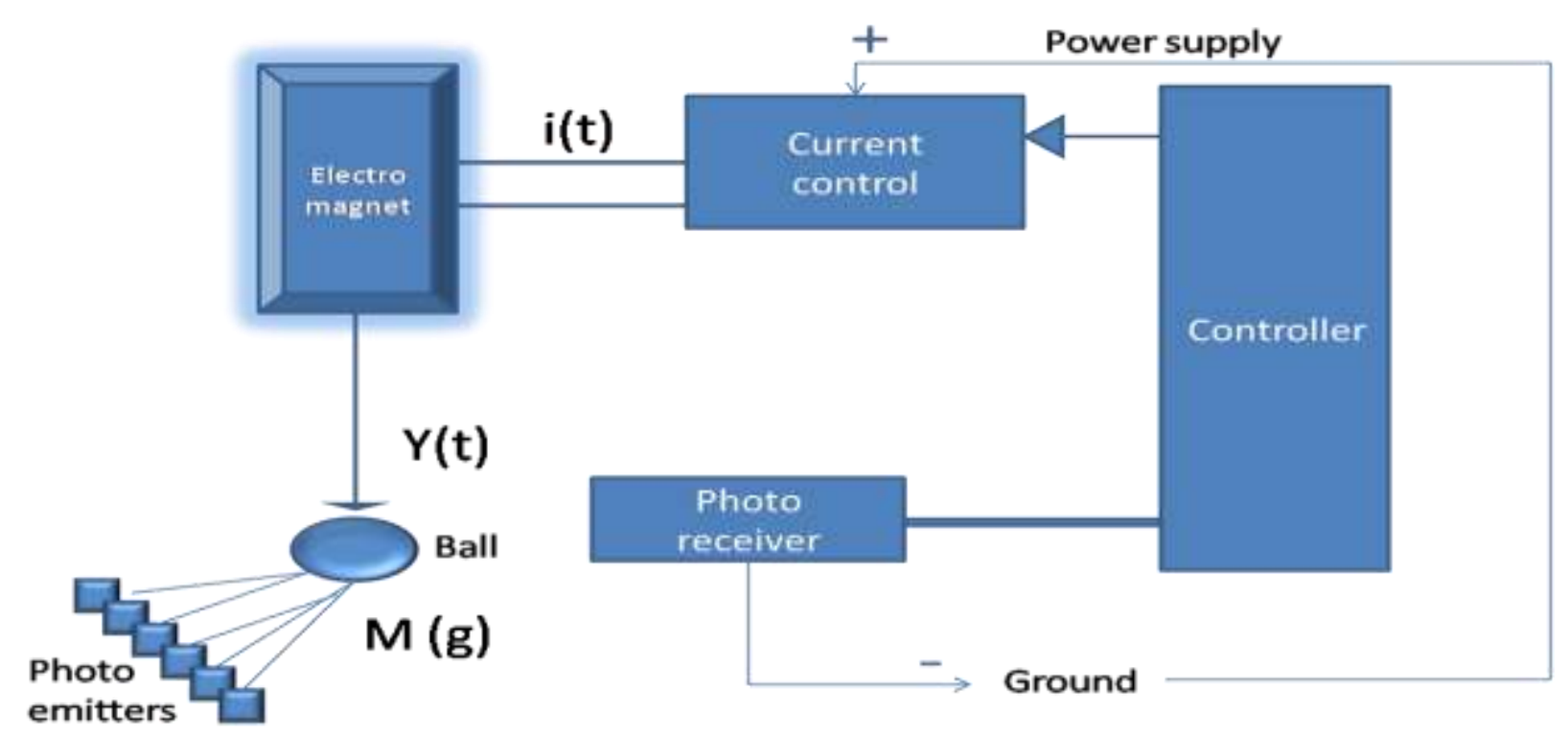

Figure 1: maglev ball system

As shown in the figure 1: the ferromagnetic ball of $\mathrm{M}$ is positioned under the electromagnet at $\mathrm{y}(\mathrm{t})$ distance. The neuro controller regulates current $\mathrm{i}(\mathrm{t})$ that flows into the coil to generate magnetic force $\alpha$ that attracts the ball. The dynamic motion of the ball guided by the law of motion (see equation 1) is as a result of the net force between the force of gravity $g$ and electromagnetic force $\alpha$.

\section{$d^{2} y(t) d t^{2}=-g+\alpha{ }^{2} i^{2}(t) y(t)-\beta M d y(t) d t$}

IV. SYSTEM DESIGN AND IMPLEMENTATION

This section will detail the steps involves in the development of the control system, starting with the system identification; then training and the
The parameter $\beta$ is a viscous friction coefficient that is determined by the material in which the magnet moves. [6] Induced that if the ball is to be suspended further away from field magnet the linear mode is presented by linearizing equation 1 about a nominal point (I, y) to obtain the equation $1[6]$.

\section{equation 1}

controller performance evaluation and finally the system implementation using a simulation model.

\section{The Neural Network (Neuro) Controller}

The first step is to identify the discrete time non linear system as in equation 2; then we train a 
network that represents the forward dynamics of the system as in equation 3 . This is better $y(k+d)=N[y(k), y(k-1), \ldots, y(k-n+1), u(k), u(k-1), \ldots, u(k-n+1)]$ $\mathrm{u}(\mathrm{k})$ is the system input, and $\mathrm{y}(\mathrm{k})$ is the system output.

The reason for this controller is to transform the non-linear maglev dynamic ball into a linear dynamic using the non-linear autoregressive $\mathrm{U}(\mathrm{k}+1)=\frac{\mathrm{a}}{\mathrm{b}^{\prime}}$

where $\mathrm{a}=$

$$
\begin{aligned}
a & =y^{r}(k+d)-f[y(k), \ldots, y(k-n+1), u(k), \ldots, u(k-n+1)] \\
b & =g[y(k), \ldots, y(k-n+1), u(k), \ldots, u(k-n+1)] \text { and } \\
d & \geq 2
\end{aligned}
$$

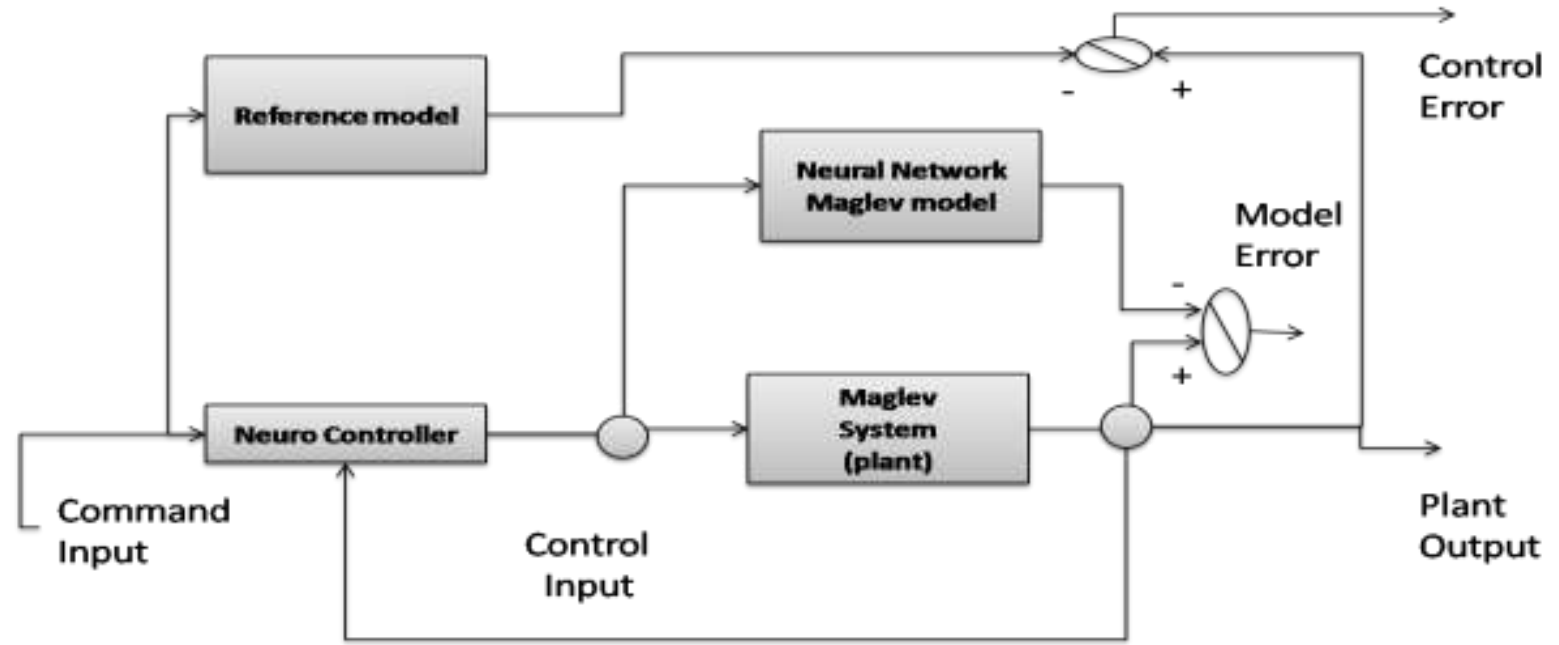

Figure 2: block diagram of the neuro controller

Now that the system is identified (equation 1) and the controller is designed (equation 2), the next step is to train the dynamic maglev ball using the magnetic position and control current which are both employ to predict the next position of the ball. This is to say that the past values of the

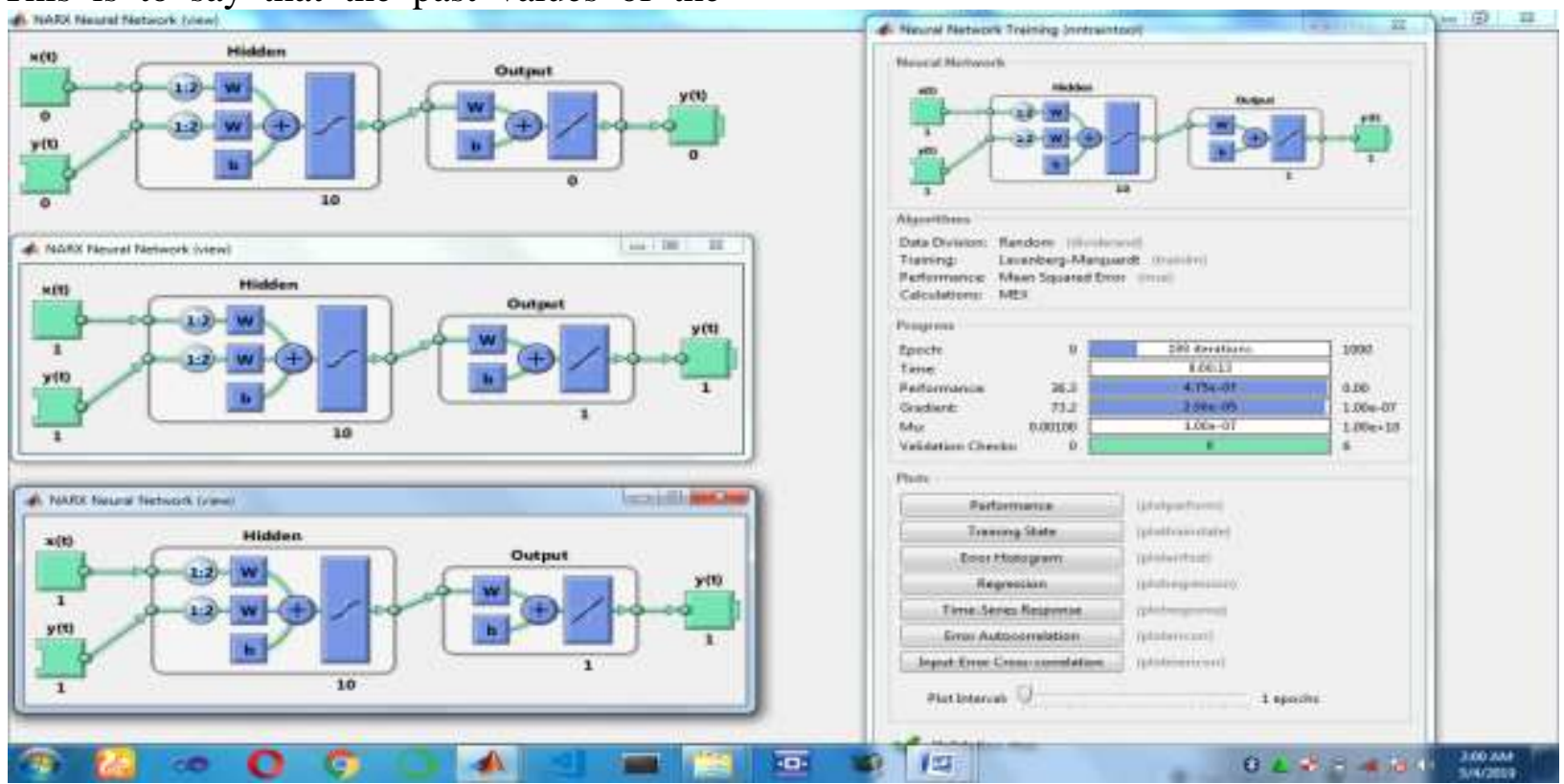

Figure 3: showing the controller and the training model described (equation 2) using the block diagram of figure 2 .

moving average model based on linear approximation model presented by [7].

\section{equation 3}


Figure 4: Neural network training regression test to evaluate and supervise the training data fitness and analyze the targeted output at a given instances depending on the validation. The response for the output element is presented in Neural network regression test was also performed (figure 5) to evaluate the response for output time series, training targets, outputs, validation targets and output, test target and output. The complete simulation model of the maglev system is presented in figure 6 , describing how the neuro controller uses controlled current and position as presented in the transfer function (figure 7) using inverse square law that reduces the electromagnetic force taken to suspend the ball. The figure 8, explains the complete simulation result of the maglev model, plotting the transfer function of the XY axes as predicted by the neural network. The blue pattern represents the actual position of the magnet while the red pattern is the predicted position by the controller.
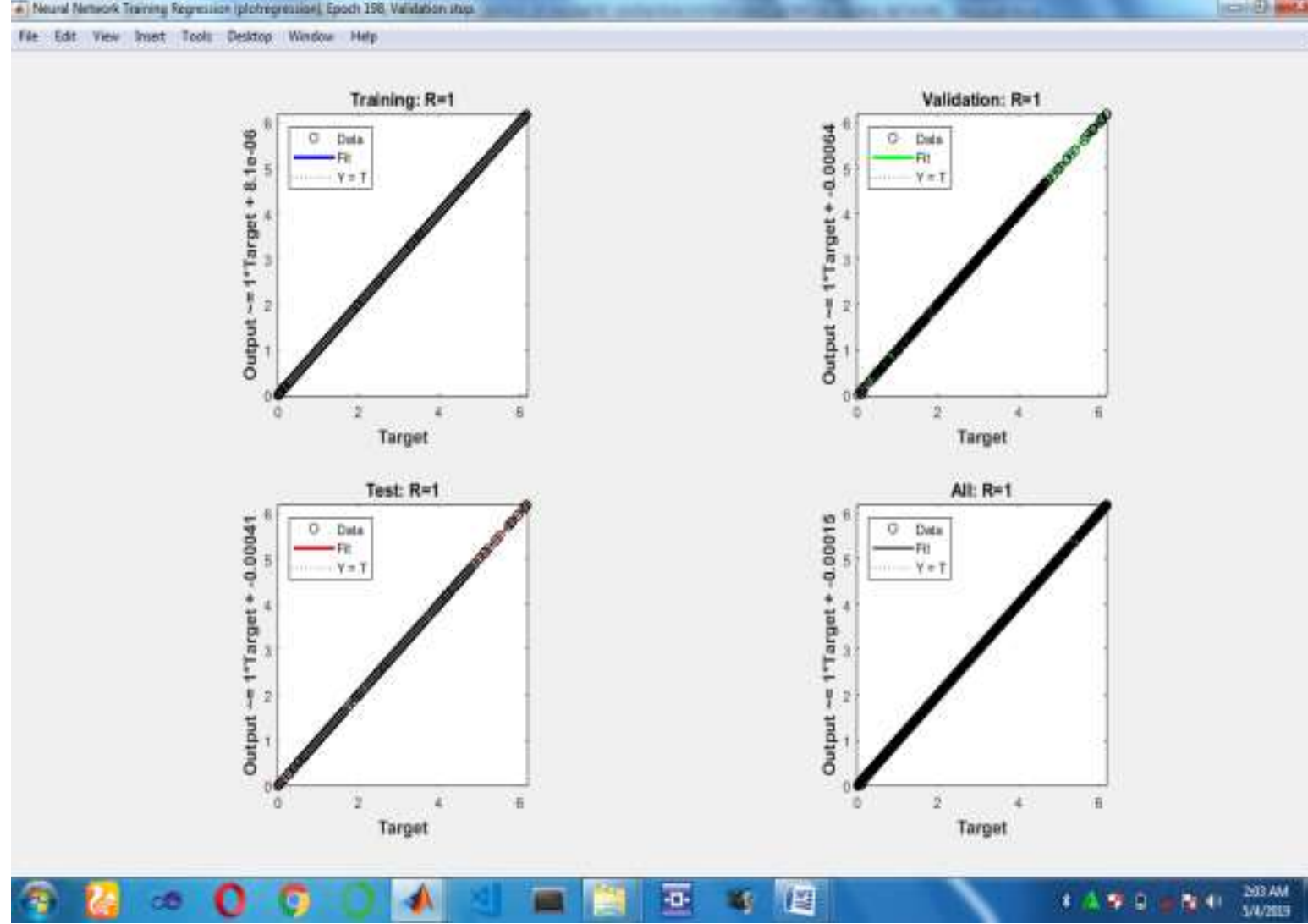

Figure 4: Regression training state of the system

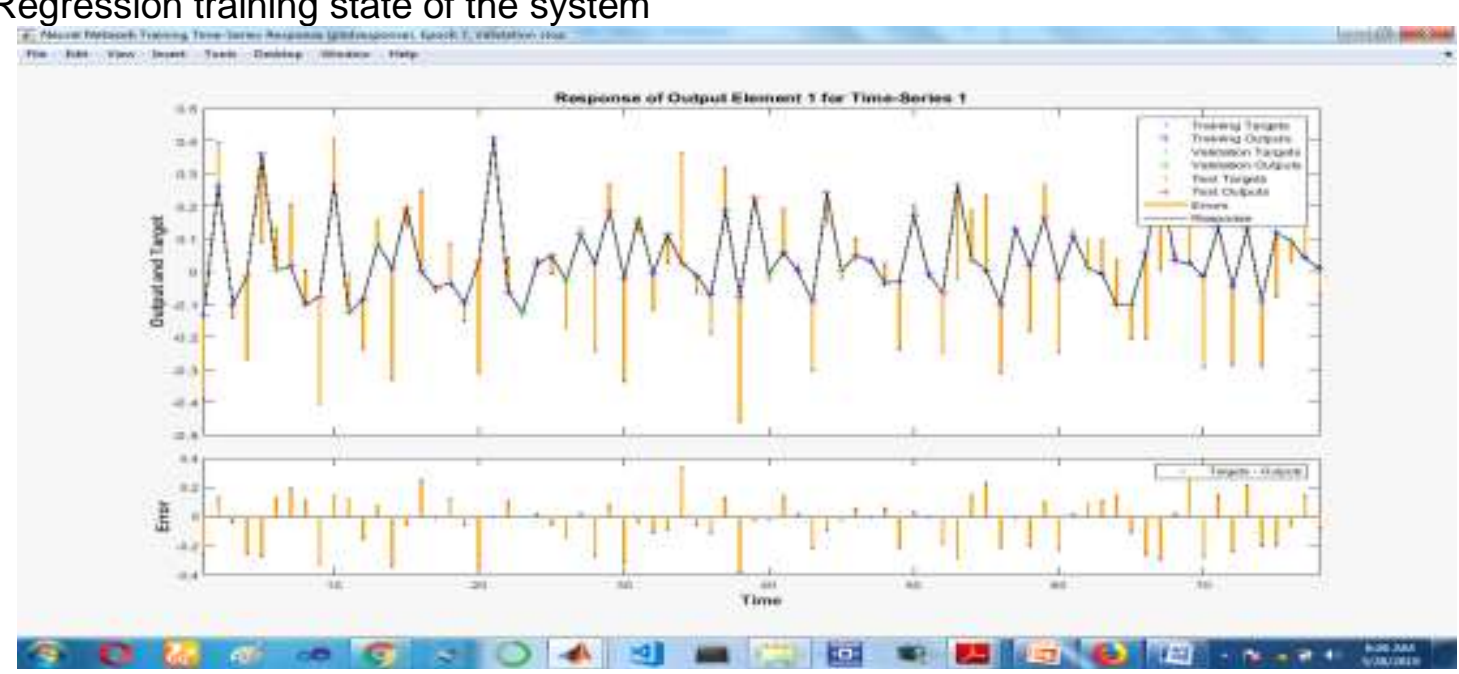

Figure 5: Response of the external output 


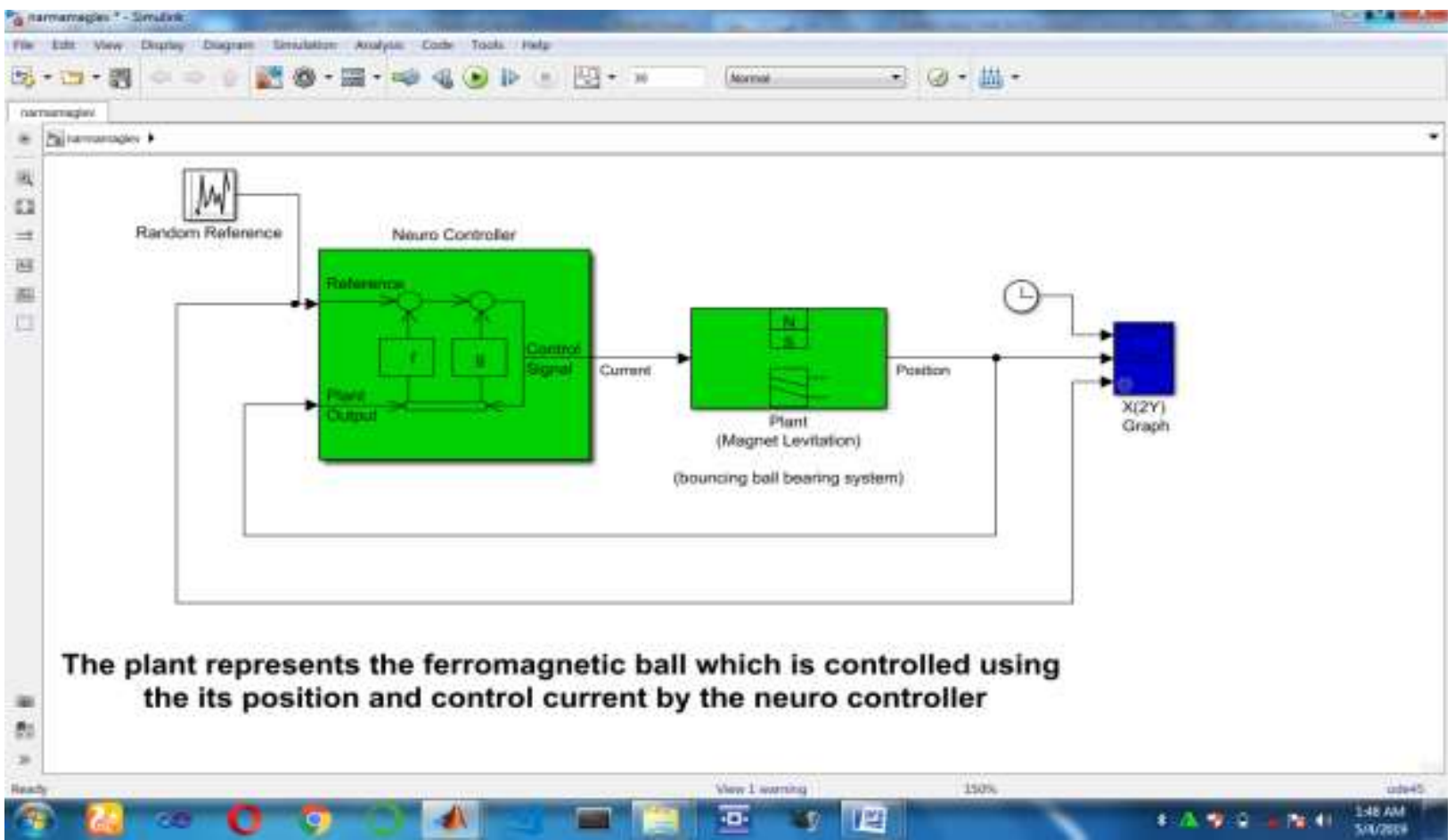

Figure 6: model of the maglev system

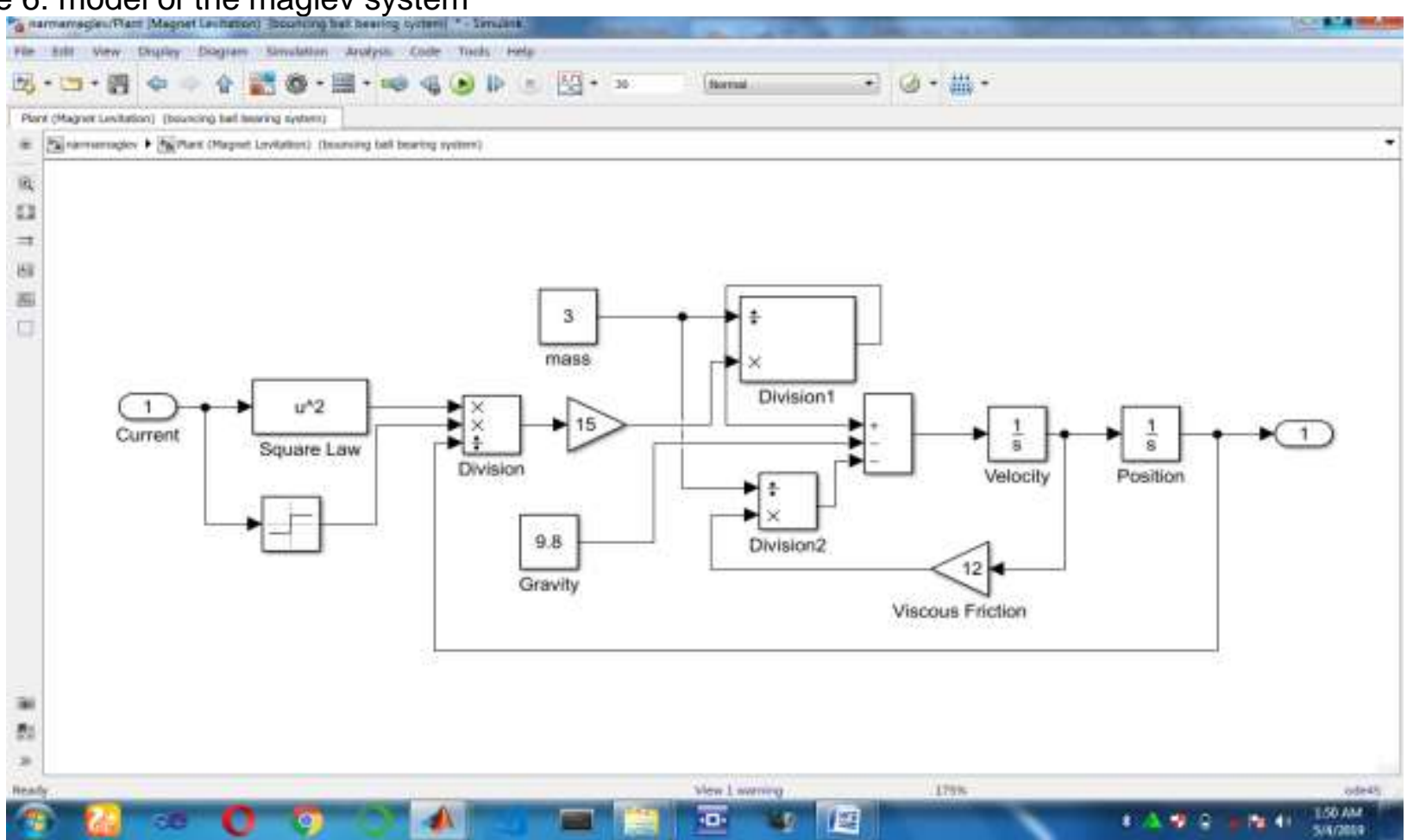

Figure 7: bouncing ball transfer function 


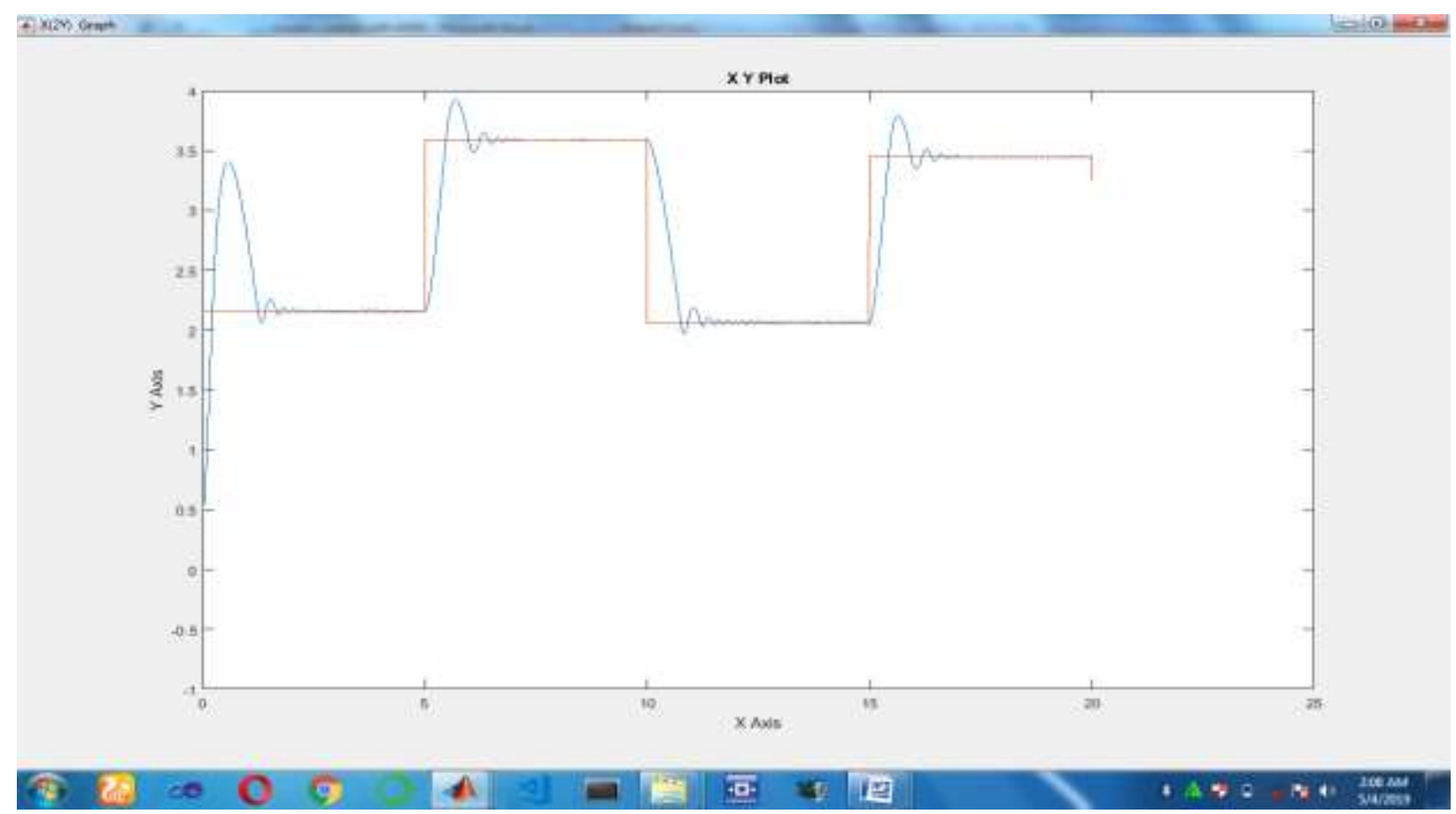

Figure 8: simulation result demonstrating the resultant controller effect on the system

\section{Conclusion}

The system reliability in most of the conventional approaches proposed for the control of non linear dynamic systems have been a major challenge, despite the huge research success recorded in various bibliographies. One of the popular systems with this time series challenge is the magnetic levitation ball, due to its dynamic bouncing nature. We have successfully presented

\section{Reference}

[1] Chaïban NASR; Neural Network Control of a magnetic levitation system; Lebanese University - Faculty of Engineering, Section IDept. of Electrical and Electronic Engineering. 2000.

[2] Valer Dolga, Lia Dolga "Modeling and Simulation of a Magnetic Levitation System" Annals Of The Oradea University. Fascicle of Management and Technological Engineering, Volume VI (XVI), 2007.

[3] Wong T. "Design of a magnetic levitation system an undergraduate project", IEEE a neuro controller designed with artificial neural network tool with the capacity to transform the non-linear maglev dynamic ball into a linear dynamic using the non-linear autoregressive moving average model based on linear approximation model. The result (figure 8) shows that even though the prediction is 25 times ahead, the result is still accurate.
Transactions on Education, pp196-200; 2010

[4] Kumar Rajesh, A.K.Ray. Artificial neural network modeling and control of Retention process in the wet end, International Journal of Information Technology and Knowledge Management July-December 2010, Volume 2, No. 2, pp. 259-264.

[5] Ying-Shing Shiao, "Design and Implementation of a Controller for a Magnetic Levitation System" Proc. Natl. Sci. Counc. ROC(D) Vol. 11, No. 2, 2011. pp. $88-94$ 
[6] Ehsan Shameli, Mir Behrad Khamesee, Jan Paul Huissoon . "Nonlinear controller design for a magnetic levitation device", Microsyst Technol 13:831-835, DOI 10.1007/s00542-006-0284-y, 2007.

[7] Narendra, Kumpati S. and Kannan Parthasarathy, "Learning Automata Approach to Hierarchical Multiobjective Analysis," IEEE Transactions on Systems, Man and Cybernetics, Vol. 20, No. 1, January/February 1991, pp. 263-272 